[Erschien in: Uwe Meixner / Albert Newen (eds): Geschichte der Ethik: Jahrbuch 6 für Philosopiegeschichte und Logische Analyse. (Paderborn: Mentis 2003), pp. 167-191].

\title{
John Stuart Mills Argument für den Utilitarismus
}

\section{Ein plausibler Weg zwischen Metaphysik und Nihilismus?}

\author{
Olaf Müller
}

\section{GLIEDERUNG}

I. Der Stein des Anstoßes

II. Vorüberlegungen für die Interpretation

III. Vernichtende Interpretationen

IV. Mills Bewegungen beim Beschreiben

V. Zwei psychologische Voraussetzungen

VI. Der Übergang in die Moral und drei Fehlschlüsse

VII. Zwischen Nihilismus und Metaphysik

Literatur

Anmerkungen

ZUSAMMENFASSUNG. Worin besteht Mills Argument für den Utilitarismus? Im psychologischen Teil des Arguments plädiert Mill für eine aggregierte Beschreibung unserer hedonistischen Werte ("Das allgemeine Glück ist ein Gut für die Gesamtheit aller Personen"). Von hier aus steuert er im normativen Teil des Arguments auf eine aggregierte Bewertung zu ("Das allgemeine Glück ist ein Gut"). Mills Übergang von Beschreibung zu Wertung beruht auf zwei versteckten Annahmen: Die erste sagt (gegen den Nihilisten), dass es Werte gibt; die zweite sagt (gegen Wert-Metaphysiker), dass die Antwort auf die Frage nach Werten nicht völlig unabhängig von dem sein kann, was wir alle de facto für wert erachten. Diese beiden Voraussetzungen scheinen keine inhaltlichen Aussagen zur Moral zu enthalten. Doch wer sie teilt, wird von Mills Argument überraschend weit in die Gefilde der Moral getrieben. Sein Argument ist besser, als die meisten Interpreten glauben.

ANMERKUNG. Dieser elektronische Text wird hier nicht in der Form wiedergegeben, in der er auf Papier erschienen ist. Zwar gibt es keine wesentlichen inhaltlichen Unterschiede zwischen den beiden Erscheinungsformen des Aufsatzes, wohl aber Unterschiede in Orthographie, Paginierung und Layout. Die Abschnitts-Nummern der Papierfassung und der elektronischen Fassung sind identisch, eignen sich also zum Zitieren. 


\title{
John Stuart Mills Argument für den Utilitarismus ${ }^{1}$
}

\section{Ein plausibler Weg zwischen Metaphysik und Nihilismus?}

\author{
Olaf Müller
}

\section{Der Stein des Anstoßes}

Kaum eine Passage aus der philosophischen Weltliteratur hat ihrem Autor stärker geschadet als der dritte Absatz des vierten Kapitels aus John Stuart Mills kleiner Schrift Utilitarianism. Mill wollte mit dieser Schrift den Utilitarismus verteidigen: Das ist die Lehre, der zufolge der moralische Wert einer Handlung einzig und allein davon abhängt, ob die Handlung geeignet ist, das allgemeine Glück unter den Menschen zu mehren. In den ersten drei Kapiteln seiner Schrift entfaltet Mill diese Lehre; im vierten Kapitel versucht er, sie zu beweisen. Und der erwähnte dritte Absatz dieses vierten Kapitels scheint den entscheidenden Schritt des Beweises zu enthalten. Er gilt als Fundgrube für besonders banale Fehlschlüsse. Hören wir Mill selbst:

The only proof capable of being given that an object is visible, is that people actually see it. The only proof that a sound is audible, is that people hear it: and so of the other sources of our experience. In like manner, I apprehend, the sole evidence it is possible to produce that anything is desirable, is that people do actually desire it. [...] No reason can be given why [5] the general happiness is desirable, except that [1] each person, so far as he believes it to be attainable, desires his own happiness. This, however, being a fact, we have not only all the proof which the case admits of, but all which it is possible to require, that [4] happiness is a good: that [2] each person's happiness is a good to that person, and [3] the general happiness, therefore, a good to the aggregate of all persons. Happiness has made out its title as one of the ends of conduct, and consequently one of the criteria of morality (Mill [U]:234 (IV, §3); Mills Kursivdruck; meine Unterstreichungen und Numerierungen).

Den schärfsten Protest haben die ersten drei Sätze dieses Gedankenganges auf sich gezogen. Offenbar lässt sich Mill von oberflächlichen Übereinstimmungen zwischen den englischen Ausdrücken

visible, audible, desirable,

auf Abwege führen. Zwar stimmt das Verhältnis zwischen

sichtbar sein und gesehen werden

mit dem Verhältnis zwischen

hörbar sein und gehört werden 
überein; der sprachoberflächlichen Parallele von "visible" und "audible" entspricht eine Parallele in der Sache. Aber diese Parallele überträgt sich nicht auf das Verhältnis zwischen

erstrebenswert sein und angestrebt werden. ${ }^{2}$

Etwas ist nicht schon erstrebenswert, wenn es bloß angestrebt wird oder angestrebt werden kann - sondern wenn es angestrebt werden sollte. ${ }^{3}$ Genau dieser wertende Beiklang geht den Wörtern "visible" und "audible" ab: Etwas ist sichtbar, wenn es gesehen werden kann - unabhängig davon, ob es gesehen werden sollte; und ganz entsprechend steht es mit dem Hörbaren.

Waren Mill diese Binsenweisheiten unbekannt? Hat er wirklich den Unterschied zwischen wertenden Wörtern wie "desirable" und beschreibenden Wörtern wie "visible" übersehen - bloß weil ihre englischen Endungen gleich klingen?

Prominente Kritiker Mills wie Moore und Bradley beantworten solche Fragen unerschrocken mit Ja. Dieser vernichtenden Interpretation beabsichtige ich entgegenzutreten. Sie ist nicht nur unplausibel, weil sie Mill einen Denkfehler zuschreibt, der schlimmer kaum sein könnte. Sie ist auch deshalb unplausibel, weil sich in ihrem Lichte der gesamte Gedankengang Mills in heillose Widersprüche verwickelt; das werde ich - nach Vorüberlegungen zur Methode meiner Interpretation (Abschnitt II) - im übernächsten Abschnitt III auseinandersetzen.

In den dann folgenden drei Abschnitten IV bis VI werde ich meine eigene Interpretation entwickeln. Meiner Ansicht nach sollte man sich vom Beginn der zitierten Passage nicht so sehr schockieren lassen, dass man ihren eigentlich argumentierenden Teil nicht mehr zur Kenntnis nimmt. Im Unterschied zu den meisten Interpreten finde ich: Mill bereitet den Übergang von Beschreibung zu Wertung sorgfältig und sehr langsam vor. Den heiklen Schritt in die Moral vollzieht Mill viel später, als all die voreiligen Interpreten gedacht haben.

Zwar wird es mir nicht gelingen, aus Mills Gedankengang einen zwingenden, lückenlosen Beweis herauszuholen. (Wie sollte solch ein Beweis auch aussehen?) Aber die Lücken, die ich in unserer Passage diagnostizieren werde, sind Lücken, mit denen man leben kann. Einige dieser Lücken lassen sich durch psychologische Zusatzannahmen füllen, für die sich Mill außerhalb unserer Passage tatsächlich ausspricht (Abschnitt V); sie haben noch nichts mit dem heiklen Übergang in die Moral zu tun. Diesen Übergang in die Moral werden wir im Abschnitt VI unter die Lupe nehmen: Argumentative Lücken lassen sich darin überraschenderweise nicht auf den 
ersten Blick dingfest machen. Aber im letzten Abschnitt VII werden wir doch noch auf eine entscheidende Lücke in dem Übergang stoßen: Ohne Begründung setzt Mill voraus, dass es erstrebenswerte Güter gibt und dass diese Güter der menschlichen Erkenntnis zugänglich sind. Wie wir sehen werden, wählt Mill mit dieser (keineswegs vollständig wertfreien) Voraussetzung einen plausiblen (wenn auch nicht zwingenden) Mittelweg zwischen einem Nihilismus der Werte und deren metaphysischer Überhöhung. Diese Voraussetzung wirkt anspruchslos (da sie ja keine spezifischen Wertungen oder Normen $\mathrm{zu}$ enthalten scheint); und es ist überraschend, dass sie trotzdem stark genug ist, um Mills Übergang von rein psychologischen Faktenbehauptungen zur utilitaristischen Wertlehre abzusichern.

Selbst wenn sich unsere Passage durch meine Rekonstruktion alles in allem vernünftiger anhören sollte als in vielen anderen Rekonstruktionen, wird am Ende unseres Weges kein Plädoyer für den Utilitarismus herauskommen. Ich werde zum Ausklang darauf bestehen, dass sich die Vielfalt der moralischen Probleme nicht auf die utilitaristische Dimension verengen lässt. Mills Fehler liegt allerdings nicht in den Überlegungen, mit denen wir uns hier beschäftigen werden, sondern in überzogenen Schlussfolgerungen, die er aus diesen Überlegungen glaubt ziehen zu können.

\section{Vorüberlegungen für die Interpretation}

Bevor wir uns in die zitierte Passage vertiefen, möchte ich einen gutgemeinten Beschwichtigungsversuch zurückweisen. Meiner Ansicht nach sollte man Mills Formulierungen nicht dadurch $\mathrm{zu}$ entschuldigen suchen, dass man an den populären Publikationsort erinnert, in dem die Schrift gleichsam wie ein Fortsetzungsroman zuerst erschienen ist: Fraser's Magazine for Town and Country - das war in der Tat eine Art Mischung aus ZEIT und Geo (ohne Bilder). Im Umfeld von Mills Kapiteln finden sich Artikel wie:

On the Education of Girls (by a Utopian), Oktober 1866;

Recollections of Ceylon: Its Forests and its Pearl Fishery, Dezember 1860;

The Horse and its Rider, Januar 1861.

Meiner Ansicht nach sprechen diese sehr britischen Themen ringsum nicht dagegen, Mills Worte ernstzunehmen. Mill hat seinen Text in den zehn Jahren nach dem ersten Erscheinen 1861 vier Mal als Monographie auf den Markt gebracht und dafür jedesmal leicht überarbeitet. Zwar wendet sich Mill ganz sicher nicht ausschließlich an Berufsphilosophen. Aber der Text ist 
sorgfältig genug durchkomponiert, um eine genaue Analyse zu verdienen. ${ }^{4}$ Wenn man die Rhetorik aus unserer Passage abzieht, bleiben genug Argumente übrig, deren Erwägung sich lohnen wird.

Dabei brauchen wir uns natürlich nicht sklavisch an Mills Text zu halten. Mill hätte Verbesserungen seiner eigenen Formulierungen begrüßt. Dafür gibt es einen Beleg, der mit den anstößigen ersten drei Sätzen aus unserer Passage zu tun hat: Die missliche Parallelisierung zwischen visible, audible und desirable hatte Theodor Gomperz, den Herausgeber der deutschen MillÜbersetzungen, so sehr gestört, dass er die Angelegenheit brieflich als "Stolperstein" bezeichnete. Er schrieb Mill im März 1868, er bedauere, dass Mill diesen Stolperstein - entgegen einem früheren Versprechen - auch aus der neuesten englischen Ausgabe nicht entfernt habe; obwohl es weit davon entfernt sei, wirke Mills Argument wie Wortklauberei, und es habe überdies den schwerwiegenden Nachteil, äußerst unübersetzbar zu sein. ${ }^{5}$ Mill antwortet am 23.4.1868:

With regard to the passage you mention in the Utilitarianism I have not had time regularly to rewrite the book, \& it had escaped my memory that you thought that argument apparently though not really fallacious which proves to me the necessity of, at least, further explanation \& development. I beg that in the translation you will kindly reserve that passage to yourself, \& will remove the stumbling block, by expressing the real argument in such terms as you think will express it best ([LLoJ]:1391).

Die deutschen Übersetzer haben sich nicht getraut, Mills Bitte nachzukommen. ${ }^{6}$ Wir werden etwas mutiger sein und versuchen, das Argument so umzuformulieren, dass es plausibler wird. Gravierende Eingriffe in den Wortlaut sind meiner Ansicht nach nicht nötig. Es wird genügen, die Reihenfolge der Sätze aus Mills Passage umzustellen.

Wie Mills wirkliches Argument im Detail aussieht, ist nicht ganz leicht zu sehen, aber in groben Zügen scheint die Sache klar. Mill möchte in der Passage von seiner deskriptiven Behauptung:

[1] Jeder Mensch strebt nach seinem eigenen Glück,

zu folgender Wertung vorstoßen:

[5] Das allgemeine Glück ist erstrebenswert. ${ }^{7}$

Selbst wenn ihm dieser Vorstoß gelingen sollte, wäre er noch nicht am Ziel. Er möchte nicht nur zeigen, dass das allgemeine Glück ein Wertmaßstab des menschlichen Handelns sein sollte; er möchte zeigen, dass das allgemeine Glück der einzige Wertmaßstab des menschlichen Handelns sein sollte. Zwar sagt Mill zu diesem ambitionierteren Ziel in unserer Passage nichts. Aber 
unmittelbar im Anschluss stellt er heraus, dass er sein ambitionierteres Ziel auf demselben Weg erreichen will wie das bescheidenere Ziel in der zitierten Passage - allerdings von einem anderen Ausgangspunkt. Statt, wie in unserer Passage, aus:

[1] Jeder Mensch strebt nach seinem eigenen Glück, abzuleiten, dass das allgemeine Glück eines der Ziele menschlichen Handelns sein sollte, will er - ganz parallel - aus der schärferen Voraussetzung:

$\left[1^{\mathrm{p}}\right] \quad$ Jeder Mensch strebt nur nach seinem eigenen Glück,

ableiten, dass das allgemeine Glück das einzige Ziel menschlichen Handelns sein sollte. ${ }^{8}$

Dass Mill auf diesen parallelen Gedankengang setzt, wird uns das Verständnis unserer Passage erleichtern. Denn zu jeder Behauptung Mills aus unserer Passage, etwa:

[2] Das Glück ist ein Gut für jeden einzelnen Menschen,

lässt sich eine parallele Behauptung konstruieren, die Mill seinem ambitionierteren Ziel zuliebe gleichfalls akzeptieren muss, in diesem Fall also:

$\left[2^{\mathrm{p}}\right] \quad$ Das Glück ist das einzige Gut für jeden einzelnen Menschen. ${ }^{9}$

Und das bedeutet, dass wir einzelne Sätze aus unserer Passage nur so interpretieren dürfen, dass sich auch deren jeweilige Parallele, wenn entsprechend interpretiert, in das Gesamtbild einfügen lässt. Damit haben wir ein scharfes Kriterium, um verschiedene Interpretationsvorschläge zu testen. Wie wir im nächsten Abschnitt sehen werden, überstehen die vernichtenden Interpretationen der Mill-Gegner diesen Test nicht.

\section{Vernichtende Interpretationen}

Die einflussreichste vernichtende Kritik des Beweises geht auf G.E. Moore zurück. (Sie beruht allerdings auf derselben Interpretationsidee, die schon Bradley verfolgt hat; dazu mehr am Ende dieses Abschnittes). Moore sieht bei Mill einen Fehlschluss, den er als "naturalist fallacy" bezeichnet. Was genau Moore unter einem naturalistischen Fehlschluss versteht, ist nicht unumstritten. Am ehesten dürfte Moore jene Verwechslung im Auge gehabt haben, von der schon die Rede war. Moore wirft Mill vor, das Erstrebenswerte mit dem tatsächlich Angestrebten zu identifizieren. Hätte Moore damit recht, so liefe Mills Beweis wie folgt. ${ }^{10}$ 
Menschen streben nach Glück. (Psychologische Voraussetzung).

"Erstrebenswert sein" und "angestrebt werden" bedeuten dasselbe. (Semantische Voraussetzung).

Also: Glück ist erstrebenswert. (Schluss aus den beiden Voraussetzungen).

Also: Glück ist ein Gut. (Schluss aus dem vorausgegangenen Satz).

Natürlich wäre solch ein Beweis alles andere als überzeugend. Denn seine semantische Voraussetzung ist falsch. Derartige Voraussetzungen sind immer falsch. Sie fallen einem Argument zum Opfer, das Moore erfunden hat - dem Argument von der Offenen Frage:

Wer zugibt, dass eine bestimmte Sache (wie etwa das Glück) angestrebt wird, der kann sich immer noch fragen: "Ist diese Sache denn auch erstrebenswert?" Die Frage wäre aber witzlos, d.h. sie wäre nicht mehr offen, wenn angestrebt werden und erstrebenswert sein dasselbe bedeuteten. In diesem Fall müsste die Frage so verrückt sein wie folgendes:

Er ist ein unverheirateter Mann im heiratsfähigen Alter, der noch nie verheiratet war - aber ist er auch ein Junggeselle?

Diese Frage ist verrückt, weil "Junggeselle" dasselbe bedeutet wie:

unverheirateter Mann im heiratsfähigen Alter, der noch nie verheiratet war.

Da hingegen die Frage:

Das und das wird angestrebt - aber ist es auch erstrebenswert?

kein Stück verrückt ist, sondern ein lohnender Gegenstand philosophischer Reflexion, kann "angestrebt werden" nicht dasselbe bedeuten "erstrebenswert sein". Und damit bricht die semantische Voraussetzung des kleinen Beweises zusammen, den Moore aus Mills Text herausgelesen hat. ${ }^{11}$ Kein Grund für Mill, alarmiert zu sein. Er hat nirgends behauptet, dass "angestrebt werden" dasselbe bedeutet wie "erstrebenswert sein". Hat er das vielleicht irgendwo versteckt vorausgesetzt? Zwei Indizien scheinen zunächst dafür zu sprechen.

Erstens bewegt sich Mill in seinem Beweis von Behauptungen über das, was angestrebt wird, zu Behauptungen über das Erstrebenswerte. Zweitens behandelt Mill das Erstrebenswerte genau so wie das Sichtbare und das Hörbare. Dies zweite Indiz ist nicht überwältigend. Mill sagt nicht, wie weit die Parallele zwischen Sichtbarem (bzw. Hörbarem) und Erstrebenswertem reicht; ob die Parallele bis in die Semantik reicht, lässt er offen. ${ }^{12}$ Doch diese Feststellung genügt nur für ein Unentschieden. Wer das Indiz aushebeln will, muss deutlicher sagen, wohin Mill die Parallele zwischen Sichtbarem und 
Erstrebenswertem führen will; wir werden darauf im letzten Abschnitt zurückkommen. Zuvor wollen wir uns klarmachen, warum auch das erste Indiz nicht für Moores vernichtende Interpretation spricht.

Es ist zwar richtig, dass sich Mill irgendwo in seinem Gedankengang von Behauptungen über das Angestrebte $\mathrm{zu}$ Behauptungen über das Erstrebenswerte bewegt. Aber diese Denkbewegung muss nicht auf der viel zu starken semantischen Voraussetzung beruhen, dass "angestrebt werden" und "erstrebenswert sein" dasselbe bedeuten. Eine solche Voraussetzung würde Mill mehr geben, als er haben will.

Einerseits würde sie seinen Gedankengang in einen zwingenden Beweis verwandeln - doch Mill sagt mehrmals, dass er keinen Beweis im üblichen Sinn des Wortes vorbringen möchte. Statt seine argumentativen Karten zu überreizen, beansprucht er in aller Bescheidenheit, die Sache des Utilitarismus plausibel zu machen; er besteht nur darauf, dass die Entscheidung für den Utilitarismus nicht einfach der Willkür anheimzustellen sei. ${ }^{13}$

Nun ist die semantische Voraussetzung, die Moore aus Mills Gedankengang herauszulesen meint, nicht allein deshalb überzogen, weil sie Mill stärkere Evidenzen in die Hand spielen würde, als er haben möchte. Sie ist, andererseits, auch deshalb überzogen, weil sie ihm mehr Behauptungen aufbürden würde, als ihm lieb sein kann. Denn die semantische Voraussetzung beweist zu viel. Würde Mill voraussetzen, dass "angestrebt werden" und "erstrebenswert sein" dasselbe bedeuten, so würden sich seine Behauptungen an allen Ecken und Enden des Gedankengangs verdoppeln: Wo er vom Angestrebten spricht, hätte er sich automatisch eine Zwillingsbehauptung über das Erstrebenswerte eingehandelt; und umgekehrt, wo er vom Erstrebenswerten spricht, hätte er sich automatisch eine Zwillingsbehauptung über das Angestrebte eingehandelt.

Doch die meisten dieser zusätzlich eingehandelten Zwillingsbehauptungen widersprechen Mills tatsächlichen Behauptungen - mit einer Ausnahme: An einer entscheidenden Stelle geht Mill vom Angestrebten zum Erstrebenswerten über; genau dort ist er auf das Zwillingspaar der betreffenden Behauptungen festgelegt. Aber diesen Übergang vollzieht Mill nur an einer einzigen Stelle. ${ }^{14}$ Wir werden diese Stelle noch genauer $\mathrm{zu}$ markieren haben. Bevor wir sie genauer markieren, möchte ich die Stellen durchgehen, an denen Mill den fraglichen Übergang gerade nicht vollzieht und die Stellen, an denen er diesen Übergang auch nicht vollziehen darf. 
Zuallererst fällt auf, dass Mill den Übergang nicht beim Glück des einzelnen vollzieht. Nirgends in seinem Text finden wir einen Schnell-Schluss der folgenden Form:

[1] Der einzelne strebt nach seinem eigenen Glück.

[-1] Also ist das Glück des einzelnen erstrebenswert. ${ }^{15}$

Meiner Ansicht nach hat Mill mit guten Gründen auf diesen Schnell-Schluss verzichtet. Das ergibt sich zumindest aus dem Testverfahren zur Ausschaltung unplausibler Interpretationen, das wir eingangs (im Abschnitt II) entwickelt haben. Wenn Mill in unserer Passage abgeleitet hätte, dass das Glück des einzelnen erstrebenswert sei, dann müsste er im ambitionierten Parallelstrang seines Gedankengangs auch folgende weitergehende Behauptung akzeptieren:

$\left[-1^{\mathrm{p}}\right] \quad$ Nichts anderes als das Glück des einzelnen ist erstrebenswert.

Aber diese Behauptung passt nicht zu Mills Ziel. Er möchte auf eine positive Wertung des allgemeinen Glücks zusteuern, und genau nicht auf eine positive Wertung des Glücks des einzelnen. (In der Interpretation, für die ich plädieren werde, erreicht Mill die positive Wertung des allgemeinen Glücks, ohne zuvor die positive Wertung des individuellen Glücks zu begründen). Mill behauptet:

[5] Das allgemeine Glück ist erstrebenswert.

Wenn nun, wie Moore annimmt, für Mill das Erstrebenswerte mit dem Angestrebten semantisch zusammenfiele, dann müssten wir ihm auch folgende Meinung zuschreiben:

[-2] Jeder erstrebt das allgemeine Glück. ${ }^{16}$

Doch auch an dieser Stelle hätte Mill das Wechselspiel zwischen Angestrebtem und Erstrebenswertem ganz bestimmt nicht zugelassen. Er meint nicht, dass jeder de facto das allgemeine Glück anstrebt (was ja auch völlig haltlos wäre), sondern dass (fast) jeder de facto das eigene Glück anstrebt. ${ }^{17}$

Man mag fragen: Warum sollten wir Mill nicht beide Meinungen zusammen zugestehen? Warum sollte Mill nicht meinen, dass jedermann sowohl das eigene Glück als auch das allgemeine Glück anstrebt? 
In der Tat, Bradley hat Mill ganz genau so verstanden. Schlimmer, Bradley hat behauptet, dass Mill den Fehler begangen habe, aus

[1] Jeder erstrebt das eigene Glück, zu folgern:

[-2] Jeder erstrebt das allgemeine Glück.

Wenn man seinem Gegner solche abenteuerlichen Folgerungen zuschreiben kann, dann hat man natürlich leichtes Spiel. Sehen wir uns einen Auszug aus Bradleys Spott an:

And yet there is a certain plausibility about it. If many pigs are fed at one trough, each desires his own food, and somehow as a consequence does seem to desire the food of all; and by parity of reasoning it should follow that each pig, desiring his own pleasure, desires also the pleasure of all. But as this scarcely seems conformable to experience, I suppose there must be something wrong with the argument, and so likewise with the argument of our philosopher (Bradley [ES]:113).

Wir brauchen uns hier nicht den Kopf darüber zu zerbrechen, inwiefern das Argument beim Schweinefutter plausibel ist und warum es sich nicht in ein paralleles Argument über das Vergnügen der Schweine verwandeln lässt. ${ }^{18}$ Denn kein vergleichbares Argument findet sich bei Mill.

Wie konnte Bradley der Idee verfallen, dass Mill ein solches Argument vertreten haben soll? Vermutlich ist Bradley über folgenden Satz bei Mill gestolpert:

No reason can be given why the general happiness is desirable, except that each person, so far as he believes it to be attainable, desires his own happiness.

Wenn man bei der Lektüre dieses Satzes wieder nicht sorgfältig zwischen Mills Behauptungen über das Angestrebte und denen über das Erstrebenswerte unterscheidet, dann erhält man in der Tat die Art von Unsinn, die Bradley bei Mill ausgemacht haben will. Aber dieser Unsinn spricht nicht gegen Mills Überlegung; er spricht gegen die unsinnige Interpretation durch Bradley und Moore.

Es lohnt sich nicht, diese Interpretation weiterzuverfolgen. Wir haben, hoffe ich, deutlich genug gesehen, dass sich Mills Gedankengang nicht angemessen verstehen lässt, wenn man annimmt, er habe das Erstrebenswerte mit dem Angestrebten identifiziert. Trotzdem mag man fragen, wie eine vernünftige Lesart des eben zitierten Satzes aussehen soll, über den Bradley gestolpert ist. Mit dieser Frage werden wir unsere Suche nach einer angemesseneren Interpretation einleiten. 


\section{Mills Bewegungen beim Beschreiben}

Wieso ergibt sich aus dem Streben des einzelnen nach seinem eigenen Glück, dass das allgemeine Glück erstrebenswert sei? Dass dieser Übergang unmittelbar einleuchtet, wird man bei aller Sympathie mit Mill kaum behaupten wollen. Meiner Ansicht nach trägt der in Rede stehende Satz keine eigene argumentative Last. Vielmehr bietet er eine Zusammenfassung oder Ankündigung des Argumentes, das noch kommen soll. Er dient der Rhetorik. Es ist nicht immer leicht, in Mills Text Argument und Rhetorik auseinanderzuhalten. Wer Mill ohne langen Atem interpretiert und überall fehlerhafte Argumente wittert, wird Mills tatsächliches Argument übersehen. Meiner Ansicht nach beginnt das Argument in unserer Passage verblüffend spät. Und erst das Ende dieses Argumentes wird uns in die Moral führen. Wir sollten uns zunächst in aller Ruhe Mills Vorbereitungen für diesen heiklen Übergang ansehen.

Wenn ich richtig liege, kommen Mills erste echte Schlussfolgerungen aus der Tatsachenbehauptung:

[1] Jeder strebt nach seinem eigenen Glück, erst mit folgenden beiden Sätzen ingang:

[2] Das Glück jeder einzelnen Person ist für diese Person ein Gut.

[3] Das allgemeine Glück ist ein Gut für die Gesamtheit der Menschen.

Mit diesen beiden Schlussfolgerungen scheint Mill wertende Aussagen zu treffen; er redet vom Glück als Gut. Aber Vorsicht, diese Annahme ist übereilt. Die Rede von Gütern in Sätzen wie [2] und [3] muss nicht wertend verstanden werden. Wenn ich sage:

Für Sokrates war die Treue gegenüber den Gesetzen Athens ein höheres Gut als das eigene Leben,

dann muss ich damit nicht unbedingt eine eigene Wertung verbinden. Im Gegenteil, der Satz könnte einer wissenschaftlichen Biographie des Sokrates entstammen und dazu dienen, die sokratischen Werthaltungen ${ }^{19}$ wertneutral beschreiben:

Sokrates strebte weniger danach, am Leben zu bleiben, als den Gesetzen Athens treu zu sein.

Wenn wir von einem Gut für jemanden sprechen, dann haben wir meiner Ansicht nach recht oft derartige Beschreibungen im Sinn. Durch die ausdrückliche Relativierung auf einen andern distanzieren wir uns gleichsam von dessen Wertung; vorsichtiger gesagt: wir deuten an, dass die fragliche Wertung auf den anderen zurückgeht und nicht unbedingt unseren eigenen 
Wertungen entspricht. Natürlich verstehen wir die Rede von einem "Gut für jemanden" oft auch wertend. Aber die wertende Lesart dieser Redeweise passt nicht zu Mills Gedankengang. Sie verleitet die Interpreten, Mills Übergang in die Moral an einer Stelle zu vermuten, wo er noch lange nicht stattfindet.

Zwei Punkte sprechen dafür, dass Mill seine Behauptung:

[2] Das Glück jeder einzelnen Person ist für diese Person ein Gut, im erläuterten, wertneutralen Sinn verstanden haben will. Erstens ergibt sich diese Behauptung dann wie von allein aus der vorangegangenen Behauptung:

[1] Jeder strebt nach seinem eigenen Glück.

Zweitens würde eine wertende Interpretation der Behauptung [2] nicht gut zum parallelen Strang aus Mills Gedankengang passen, den wir eingangs erwähnt und schon mehrmals herangezogen haben, um unplausible Interpretationen auszuschalten. Wäre Mills Behauptung:

[2] Das Glück jeder einzelnen Person ist für diese Person ein Gut, wertend $\mathrm{zu}$ verstehen, nicht beschreibend, so müsste er auch die weitergehende Behauptung aus dem Parallelstrang:

$\left[2^{\mathrm{p}}\right] \quad$ Das Glück jeder einzelnen Person ist für diese Person das einzige Gut,

wertend verstehen. Er müsste also ungefähr sagen:

Das Glück jeder einzelnen Person ist legitimerweise für diese Person das einzige Gut,

oder deutlicher:

Jeder einzelne sollte nur nach seinem eigenen Glück streben.

Diese Position lässt sich kaum mit Mills Utilitarismus vereinbaren. Der Utilitarist plädiert für das allgemeine Glück als einzigen Wertmaßstab menschlichen Handelns; dass jeder einzelne nur nach seinem eigenen Glück streben soll, ist eine andere Lehre. (Es ist die Lehre der sogenannten ethischen Egoisten).

Damit ist klar, dass Mill den Satz

$\left[2^{\mathrm{p}}\right] \quad$ Das Glück jeder einzelnen Person ist für diese Person das einzige Gut,

nur als Deskription unterschreiben darf; und diese deskriptivistische Interpretation vererbt sich auf den Satz aus unserer Passage, um den es uns 
eigentlich zu tun war. Wir mögen aus dem Ausdruck "ist ein Gut für X" noch so deutlich eine Wertung heraushören - Mill muss diesen Ausdruck ohne wertenden Beiklang verwenden, wenn er behauptet:

[2] Das Glück jeder einzelnen Person ist für diese Person ein Gut.

Wie ergibt sich aus diesem beschreibenden Satz die nächste Behauptung Mills, die wir schon angeführt haben? Das hängt davon ab, wie wir Mills nächsten Schritt verstehen:

[3] Das allgemeine Glück ist ein Gut für die Gesamtheit der Menschen.

Ist das eine Beschreibung oder eine Wertung? Müssten wir Mills neue Behauptung wertend verstehen, so ergäbe sie sich nicht so ohne weiteres aus ihrer beschreibenden Vorläuferin [2]. Dürften wir die Behauptung dagegen ebenfalls als Beschreibung verstehen, so erhöhen sich offenbar die Chancen dafür, dass Mill immer noch auf sicheren Bahnen argumentiert. ${ }^{20}$

Dieser optimistische Eindruck scheint sich zu bestätigen, wenn wir uns die Lage an einem einfacheren Beispiel verdeutlichen. Nehmen wir z.B. an, jeder Tibetaner strebe nach der Selbstbestimmung Tibets. Dann können wir diesen Sachverhalt wertneutral beschrieben, indem wir sagen:

Für die Gesamtheit der Tibetaner ist die Selbstbestimmung Tibets ein Gut.

Und diese Beschreibung folgt offenbar aus der Zusammenfassung entsprechender Beschreibungen jedes einzelnen Tibetaners:

Für jeden einzelnen Tibetaner ist die Selbstbestimmung Tibets ein Gut.

Es gibt allerdings wichtige Unterschiede zwischen dem Übergang im TibetBeispiel und Mills ähnlichem Übergang beim Glück. Bei der zusammenfassenden Schlussfolgerung aus der Einzelbeschreibung eines jeden Tibetaners haben wir nur die (prospektiven) Nutznießer des fraglichen Gutes zusammengefasst; wir sind von jedem einzelnen Tibetaner zur Gesamtheit der Tibetaner übergegangen, ohne auch das fragliche Gut - die Selbstbestimmung Tibets - eigens zusammenzufassen. Z.B. sind wir nicht von der Selbstbestimmung Tibets zur allgemeinen Selbstbestimmung Tibets übergegangen. Eine solche Zusammenfassung wäre in diesem Beispiel nicht nötig gewesen, da sich die Wünsche der Tibetaner sowieso auf ein und dieselbe Sache richten: Für den Dalai Lama ist die Selbstbestimmung Tibets dasselbe wie für den tibetischen Bettelmönch. Tibets Selbstbestimmung ist sozusagen nicht personengebunden. 
Anders beim Glück: Das Glück des Dalai Lama ist nicht das Glück irgendeines tibetischen Bettelmönchs. Darum muss Mill in seinem Argument mehr tun, wenn er den gewünschten zusammenfasssenden Übergang vollziehen will. Und in der Tat, er bewegt sich beim Übergang von Das Glück jeder einzelnen Person ist für diese Person ein Gut,

$\mathrm{Zu}$

[3] Das allgemeine Glück ist ein Gut für die Gesamtheit der Menschen, nicht nur vom einzelnen Menschen zur Gesamtheit aller Menschen, sondern auch vom Glück des einzelnen zum allgemeinen Glück.

An dieser Stelle verbergen sich logische Feinheiten, die man leicht übersieht. Die Rede vom Glück jeder einzelnen Person in [2] bietet nicht einfach nur eine andere Ausdrucksweise für die Rede vom allgemeinen Glück in [3]. Um den feinen Unterschied, auf den es hier ankommt, deutlich zu machen, wollen wir beide Sätze etwas ausdrücklicher fassen:

[2*] Für jeden Menschen X gilt: Es ist ein Gut für X, dass X glücklich ist.

[3*] Es ist ein Gut für die Gesamtheit der Menschen, dass alle Menschen glücklich sind. ${ }^{21}$

Mills Übergang ist also komplizierter als der Übergang im Falle Tibets. Und das bedeutet, dass wir uns immer noch nicht sicher sein können, ob Mills Übergang wirklich funktioniert. Um den Übergang abzusichern, muss Mill Voraussetzungen machen, die wir bislang nicht in den Blick genommen haben und die der Gegenstand des nächsten Abschnitts sein werden.

\section{Zwei psychologische Voraussetzungen}

Schlüsse von Behauptungen über Güter für jeden einzelnen zu Behauptungen über zusammengefasste Güter für alle funktionieren nicht immer. Das zeigt der folgende Fall. Zwar gilt (wie wir zum Zwecke des Argumentes annehmen wollen):

Für jeden Lottospieler ist der Hauptgewinn ein Gut. (D.h. für jeden Lottospieler X gilt: Es ist ein Gut für X, dass X den Hauptgewinn bekommt).

Aber daraus folgt noch lange nicht:

Für die Gesamtheit der Lottospieler ist der allgemeine Hauptgewinn ein Gut. (D.h. für die Gesamtheit der Lottospieler ist es ein Gut, dass alle Lottospieler den Hauptgewinn bekommen). 
Wenn alle Lottospieler einen Hauptgewinn bekommen, so gewinnt jeder viel zu wenig, und der Reiz des Lottospiels geht verloren; daran kann die Gesamtheit der Lottospieler kein Interesse haben. Der Witz des Lottospiels besteht darin, dass ein Mitspieler (oder eine kleine Gruppe der Mitspieler) genau das gewinnt, was die vielen anderen verlieren. ${ }^{22}$ Lotto ist ein Nullsummenspiel, sagen die Mathematiker. Und dieser unschöne Zug des Lottos trägt die Schuld daran, dass der eben ins Auge gefasste Übergang vom Gut für jeden einzelnen Lottospieler zum allgemeinen Gut für die Gesamtheit der Spieler nicht funktioniert. Solch ein Übergang funktioniert nie für Güter, die in Nullsummenspielen verteilt werden.

Dies Ergebnis braucht Mill noch nicht zu beunruhigen. Denn das Streben nach Glück ist ganz gewiss kein Nullsummenspiel. Es stimmt nicht, dass jeder einzelne immer nur auf Kosten der anderen glücklich werden kann. Im Gegenteil, es ist ein psychologisches Faktum, dass der einzelne tiefes Glück gewinnen kann, indem er andere glücklich macht. Anders als beim Lotto sind beim Streben nach Glück Sozialisierungs- und Kooperationsgewinne möglich.

Dass es sich so verhält, sagt Mill zwar nicht in der Passage, um die es uns hier zu tun ist. Aber erstens sagt er etwas sehr ähnliches an anderen Stellen in unserem Kapitel (dazu gleich). Und zweitens kann er ohne diese zusätzliche Voraussetzung unter keinen Umständen zum Ziel kommen. Ohne diese Voraussetzung wäre der Utilitarismus ganz unplausibel. Denn wenn das Streben nach Glück, entgegen der zusätzlichen Voraussetzung, ein Nullsummenspiel wäre, d.h. wenn jeder Glücksgewinn des einen notwendig mit einem gleich großen Glücksverlust des andern einherginge, wenn also die Summe des gesamten Glücks immer konstant bliebe: dann wäre es unmöglich, das allgemeine Glück zu mehren, und die utilitaristische Doktrin liefe ins Leere.

Damit haben wir eine versteckte Voraussetzung in Mills Gedankengang entdeckt - eine Voraussetzung, die allerdings nicht ganz unplausibel ist:

(A) Das Streben nach Glück ist kein Nullsummenspiel; Menschen sind so konstituiert, dass sie Glück aus der Beförderung des Glücks anderer ziehen können.

Dass Mill eine derartige psychologische Behauptung für richtig hält, steht außer Zweifel. ${ }^{23}$ Mill bringt sie ausgerechnet an der Stelle unseres Kapitels ins Spiel, wo er die weitgehende These verteidigen will, dass jeder ausschließlich nach seinem eigenen Glück strebt. Streben Menschen nicht manchmal z.B. nach Tugend? So lautet ein Einwand, den Mill zurückweisen möchte. ${ }^{24}$ Und er beantwortet den Einwand, indem er herausarbeitet, wie das 
Streben nach Tugend im Streben nach Glück aufgeht. Wenn sie einen entsprechenden Lernprozess durchlaufen haben, dann (meint Mill) erstreben Menschen die Tugend als Teil des Glücks. ${ }^{25}$ Und wenn man mit Mill unter Tugend die Disposition versteht, dass allgemeine Glück zu vermehren, dann ergibt sich daraus die Voraussetzung, die Mill auf jeden Fall braucht, wenn seine utilitaristische Doktrin nicht in Leere laufen soll. ${ }^{26}$

Man kann sich fragen, ob diese Voraussetzung (A) stark genug ist, um wirklich den Übergang von der Beschreibung der Werthaltungen einzelner:

[2] Das Glück jeder einzelnen Person ist für diese Person ein Gut, zur Beschreibung der Werthaltungen der Gemeinschaft abzusichern:

[3] Das allgemeine Glück ist ein Gut für die Gesamtheit der Menschen.

Vielleicht braucht Mill für seinen Übergang eine noch stärkere Voraussetzung:

(B) Menschen sind so konstituiert, dass sie Glück nur aus der Beförderung des Glücks anderer ziehen können; genauer: dass sie das ihnen wertvollste Glück nur aus der Beförderung des Glücks anderer ziehen können.

Auch diese psychologische Voraussetzung hält Mill für richtig. ${ }^{27}$ Aus welchen Gründen er sie für richtig hält, können wir hier nicht einzeln erörtern; unser Thema ist nicht Psychologie, sondern das, was man aus psychologischen Voraussetzungen am Ende für die Moral ableiten kann. Und so wollen wir uns anhand eines parallelen Beispiels klar machen, dass der gewünschte Übergang mithilfe einer à la (B) verstärkten Voraussetzung tatsächlich funktionieren würde.

Betrachten wir eine Gruppe von Polarforschern, die im ewigen Eis vom Weg abgekommen sind und die sich nur durch Kooperation aus ihrer brenzligen Lage befreien können. Für sie gilt eine Voraussetzung, die der eben verstärkten Voraussetzung für Mills Übergang strukturell gleicht:

Jeder einzelne Polarforscher kann nur überleben, wenn alle Polarforscher überleben.

Unter dieser Voraussetzung lässt sich aus der Beschreibung der Werthaltungen der einzelnen Forscher:

Für jeden Polarforscher ist sein eigenes Überleben ein Gut, eine Beschreibung der kollektiven Werthaltung ableiten:

Für die Gruppe der Polarforscher ist das kollektive Überleben ein Gut. 
In diese kollektivistische Beschreibung geht nicht nur die Summe der subjektiven Werthaltungen der einzelnen Forscher ein, sondern auch das objektive Faktum, dass die einzelnen Forscher in ihrem Streben zu überleben aufeinander angewiesen sind. Dadurch kommt sozusagen mehr Objektivität ins Spiel - aber immer noch keine Wertung.

Und ganz entsprechend kann Mill zur objektiveren Beschreibung [3] der kollektiven Werthaltung aller Menschen vordringen - wenn die genannte (recht starke) psychologische Voraussetzung zutrifft, dass Menschen ein bestimmtes, tiefes Glück (das Glück, das sie besonders hochschätzen) nur erreichen können, wenn sie anderen Menschen beim Streben nach Glück unter die Arme greifen.

Selbst wenn diese starke psychologische Voraussetzung richtig ist, sind wir noch nicht am Ziel. Den entscheidenen Schritt in die Moral haben wir weiter vor uns hergeschoben. Er ist das Thema des nächsten Abschnitts.

\section{Der Übergang in die Moral und drei Fehlschlüsse}

In den zurückliegenden beiden Abschnitten haben wir mit Mill keine einzige Werthaltung begründet; wir haben Werthaltungen bislang nur beschrieben erst individuelle Werthaltungen und dann eine kollektive Werthaltung:

[3] Das allgemeine Glück ist ein Gut für die Gesamtheit der Menschen.

Was folgt aus derartigen Beschreibungen für die Moral? Wie ergibt sich, dass wir das allgemeine Glück anstreben sollten? Dass Mills nächster Schritt in die Moral führen muss, scheint festzustehen. Wir befinden uns also an einem kritischen Wendepunkt des Gedankenganges. Um an diesem Wendepunkt nicht ins Stolpern zu geraten, sollten wir in möglichst kleinen Schritten weitergehen. Meiner Ansicht nach formuliert Mill in unserer Passage eine moralisch wertende Behauptung, die besonders eng an das bislang Erreichte anschließt. Sie lautet:

[4] Das allgemeine Glück ist ein Gut. ${ }^{28}$

Hier verwendet Mill den Begriff des Gutes eindeutig im wertenden Sinn. Denn diesmal bezieht Mill den Begriff weder auf einzelne noch auf eine Gruppe. Er spricht nicht von einem Gut für $X$ - er spricht ohne Zusatz vom Glück als Gut. Und das bedeutet, dass er hier nicht die Werthaltungen irgendwelcher anderen beschreibt, sondern dass er endlich seine eigene Werthaltung ausdrückt. 
An der sprachlichen Oberfläche wirkt Mills Schritt hin zu seiner eigenen Werthaltung harmlos. Mill lässt eine adverbiale Bestimmung weg, wenn er schließt:

Das allgemeine Glück ist ein Gut für die Gesamtheit der Menschen. Also: Das allgemeine Glück ist ein Gut.

Durch Beseitigung adverbialer Bestimmungen kann man eine ganze Reihe wasserdichter Schlüsse gewinnen; etwa:

Bertrand Russell hat den Literatur-Nobelpreis für sein Buch "Marriage and Morals" gewonnen. Also hat Bertrand Russell den Literatur-Nobelpreis gewonnen.

Solche Schlüsse sind wasserdicht, weil Wahrheiten durch Weglassen von Information zwar weniger informativ, aber dafür sicherer und jedenfalls nicht weniger wahr werden.

Aber das funktioniert nicht immer; die Beseitigung einer adverbialen Bestimmung muss nicht unbedingt auf Kosten des Informationsgehaltes gehen. In Mills Fall bedeutet die Beseitigung der adverbialen Bestimmung keinen Verlust an Information, also auch keinen Gewinn an Sicherheit. Vielmehr bewirkt die Beseitigung der Wörter

für die Gesamtheit der Menschen

aus der Beschreibung:

[3] Das allgemeine Glück ist ein Gut für die Gesamtheit der Menschen, eine Änderung der Information; die Beschreibung verwandelt sich in eine Wertung. Und bei dieser Verwandlung könnten sich Fehler einschleichen. Mills Schluss ist also nicht unbedingt wasserdicht.

Man sollte diesen Verdacht besser nicht durch Rückgriff auf Humes berühmte Sein/Sollen-Schranke durchboxen. Die Sein/Sollen-Schranke ist eine Verallgemeinerung aus einzelnen Fällen (in denen der Schluss vom Sein zum Sollen fehlschlug ${ }^{29}$ ). Falls Mills Schluss die Sein/Sollen-Schranke überwindet, so muss das nicht unbedingt gegen seinen Schluss sprechen; es könnte gegen die Sein/Sollen-Schranke sprechen.

Wenn Mills Schluss wirklich nicht wasserdicht ist, dann muss man die Lücken in seinem Schluss präzise aufdecken können; man sollte nachweisen, wie das Ergebnis des Schlusses falsch werden kann, selbst wenn seine Voraussetzungen stimmen. (Solch ein Nachweis wäre jedenfalls instruktiver als ein Rundumschlag à la Hume). Wir werden diesen Nachweis im 
vorliegenden Abschnitt ansteuern, dabei auf Schwierigkeiten stoßen und ihn erst im nächsten Abschnitt wirklich erreichen.

Man findet Lücken in irgendwelchen Beweisen üblicherweise durch Betrachtung gleichgebauter Fälle. Mills Beweis hat auf den ersten Blick eine ganze Reihe dubioser Parallelen. Wir werden im verbleibenden Teil dieses Abschnittes drei Schlüsse unter die Lupe nehmen, die so ähnlich gebaut sind wie Mills Schluss und die garantiert nicht funktionieren. Wie sich aber bei genauerem Hinsehen herausstellen wird, bieten die drei Fehlschlüsse keine exakte Parallele des Schlusses von Mill. Am Ende dieses Abschnittes wird Mills Schluss immer noch erstaunlich gut dastehen.

Hier ist unser erster Fall. Wer der Meinung ist, dass die Achtung der Athenischen Gesetze für Sokrates ein Gut (gewesen) ist, kann immer noch bestreiten, dass die Achtung der Athenischen Gesetze schlechthin ein Gut (gewesen) ist: Der Biograph des Sokrates kann die Gesetze Athens für verderbt halten; er kann sogar gegen jede Achtung von Gesetzen sein. Selbst ein gestandener Anarchist mag imstande sein, eine wahrheitsgetreue Sokrates-Biographie zu verfassen. Und schon die mögliche Existenz des anarchistischen Biographen beweist, dass man nicht von der Logik gezwungen werden kann, den folgenden Schluss zu vollziehen:

Die Achtung vor Athens Gesetzen ist für Sokrates ein Gut; also ist die Achtung vor Athens Gesetzen ein Gut.

Verläuft dieser Fehlschluss völlig parallel zu Mills Schluss? Nein: Mill wollte eine positive Wertung des allgemeinen Glücks nicht aus der tatsächlichen Werthaltung eines einzelnen ableiten, sondern aus der tatsächlichen Werthaltung eines Kollektivs. Das ist ein wichtiger struktureller Unterschied.

Wird Mills Schluss dadurch vielleicht besser? Es scheint nicht so. Auch die kollektive Fassung seines Schlusses lässt sich durch Betrachtung paralleler Schlüsse erschüttern. Das zeigt unser zweiter Fall. Hätte Mill im fernen Indien eine isolierte Meditationsgemeinschaft entdeckt, deren Mitglieder danach streben, gemeinsam auf möglichst schmerzhafte Weise und doch möglichst würdevoll zu sterben, dann hätte er folgende Beschreibung akzeptieren müssen:

Für die indische Meditationsgemeinschaft ist der gemeinsame schmerzhafte Tod in Würde ein Gut.

Aber das hätte Mill noch lange nicht dazu zwingen müssen, den Utilitarismus samt hedonistischer Wertlehre preiszugeben und stattdessen das indische Streben nach Schmerz, Tod und Würde hochzuwerten. ${ }^{30}$ Das ist 
nicht weiter überraschend. Wer, wie ein Ethnologe, von außen beschreibt, welche kollektiven Werthaltungen in irgendeiner Gemeinschaft verfolgt werden, braucht diese Werte nicht unbedingt $\mathrm{zu}$ teilen. Auch aus der Beschreibung kollektiver Werthaltungen folgt also nichts über die Richtigkeit dieser Wertungen.

Nur: Mill zeichnet das allgemeine Glück nicht von außen als ein Gut für die Gesamtheit der Menschen aus; er gehört selber zu der Gemeinschaft, deren Werthaltung er beschreibt. Er beschreibt sie von innen.

Wird dadurch der Zusammenhang zwischen beschriebener Werthaltung und eigener Wertung enger? Sicherlich ist es nicht einfach, sich von den Werthaltungen der eigenen Gemeinschaft zu distanzieren - anders als bei fremden Werthaltungen, die man wie ein Ethnologe von außen betrachtet. Kommt diesem psychologischen Punkt auch logisches Gewicht zu?

Es scheint so. Es klingt wie ein handfester logischer Widerspruch, wenn einer sagt:

Für mich und meine Gemeinschaft ist das allgemeine Glück ein Gut; aber das allgemeine Glück ist vielleicht kein Gut.

Das klingt deshalb wie ein Widerspruch, weil der Sprecher im zweiten Teilsatz die Werthaltung suspendiert, deren Vorliegen er im ersten Teilsatz behauptet. $^{31}$ Beides kann offenbar nicht in ein und demselben Atemzug richtig sein. Und das scheint zu bedeuten, dass ein Schluss nach folgendem Muster zwingend ist:

Für mich und meine Gemeinschaft ist X ein Gut; also ist X ein Gut.

Doch der Anschein trügt, wie unser dritter Fall zeigt. Ein Mitglied der vorhin betrachteten indischen Meditationsgemeinschaft ist $\mathrm{zu}$ einem Schluss nach diesem Muster nicht gezwungen. Er kann sehr wohl recht haben, wenn er (gleichsam von innen) sagt:

Für mich und meine Gemeinschaft ist ein kollektiver schmerzhafter Tod in Würde ein Gut; aber vielleicht ist ein kollektiver schmerzhafter Tod in Würde kein Gut.

Der Inder könnte durch diesen Text seine Zweifel an dem Wertsystem ausdrücken, dem er in seiner Gemeinschaft gefolgt ist. Er mag sich z.B. fragen, ob das Wertesystem seiner Gemeinschaft durch einen kompetenten äußeren Betrachter infrage gestellt und von einem überlegenen Wertsystem abgelöst werden könnte. Und eine ähnliche Frage scheint Mills Gegnern offenzustehen, selbst wenn sie zugeben, dass das allgemeine Glück für ihre Gemeinschaft bislang ein Gut gewesen ist. Wenn Mills Gegner nach diesem 
Zugeständnis immer noch fragen können, ob das gemeinsame Wertsystem mit Recht von außen überwunden werden könnte, dann weist dies auf eine Lücke in Mills Schluss hin.

Aber steht ihnen diese Frage wirklich offen? Sind die beiden Fälle wirklich parallel? Nicht ganz: In einer entscheidenden Hinsicht haben es Mills Gegner schwerer als der indische Dissident, der sich fragt, ob die Werthaltungen seiner Meditationsgemeinschaft von außen korrigiert werden könnten. Die Meditationsgemeinschaft ist von Gemeinschaften mit anderen Werten umgeben; Kritik von außen droht ihr als reale Möglichkeit. Im Gegensatz dazu streben laut Mill alle Menschen nach Glück und konstituieren die Wertung des allgemeinen Glücks, auf die er seinen entscheidenden Schritt in die Moral aufbaut. Wenn das psychologisch richtig sein sollte, dann gibt es keinen äußeren Standpunkt, von dem aus sich die kollektive Wertschätzung des allgemeinen Glücks zurückweisen ließe.

Ob Mills hedonistische Psychologie universell gültig ist und wirklich alle Menschen aus allen Zeiten und Kulturen erfasst, kann ich hier nicht erörtern. (Sie ist weniger unplausibel, als auf den ersten Blick scheinen mag, wenn man bedenkt, dass Mill einen Begriff des Glücks verficht, der recht viel umfasst, z.B. die Tugend). Im nächsten Abschnitt möchte ich Mills Psychologie zum Zweck des Argumentes akzeptieren und herausarbeiten, wie man Mills moralischen Schlüssen widerstehen kann, selbst wenn man seine universellen Beschreibungen der menschlichen Psyche für richtig hält. Wie wir sehen werden, verläuft Mills Übergang in die Moral nicht ohne Lücken; trotzdem sind die zusätzlich nötigen Voraussetzungen einigermaßen harmlos.

\section{Zwischen Nihilismus und Metaphysik}

Kann man Mill zugeben, dass das allgemeine Glück de facto psychologico ein Gut für die Gesamtheit der Menschen ist, ohne dem allgemeinen Glück einen moralischen Wert beizumessen? Kann man sogar zugeben, dass das allgemeine Glück das einzige Gut für die Gesamtheit der Menschen ist, ohne dem allgemeinen Glück einen moralischen Wert beizumessen? ${ }^{32}$ Ich sehe mindestens zwei extreme Möglichkeiten, Mills Schlussfolgerung zu vermeiden. Zum einen könnte man die Einschlägigkeit des tatsächlichen Wertmaßstabes der Menschen bestreiten, weil man meint, dass dieser einem übergeordneten Wertmaßstab weichen muss: Es gibt, könnte man sagen, höhere (oder jedenfalls andere) Güter, in deren Licht die irdischen Wertmaßstäbe null und nichtig sind. Das ist ein respektabler Gedanke, der z.B. in bestimmten Formen der Religion hochgehalten wird. Mills Wertungen entspringen dem Kreis aller Menschen. Man kann seinen 
Wertungen entgegentreten, indem man den Kreis verlässt und Werten aus dem Jenseits das Wort redet. Gegen diese Möglichkeit ist Mills Argument machtlos. Und das bedeutet, dass er für sein Argument ungefähr folgendes voraussetzen muss:

(C) Es gibt keinen vernünftigen transzendenten Standard für die Bestimmung von Gütern - keinen Standard, der nicht irgendwie mit uns Menschen zusammenhängt. ${ }^{33}$

Die Möglichkeit transzendenter oder metaphysischer Güter hatte Mill mit seinem Argument offenbar nicht ausdrücklich im Visier. Die zweite Möglichkeit, gegen die Mills Argument machtlos ist, liegt im entgegengesetzten Extrem. Dieser Möglichkeit zufolge ist das allgemeine Glück deshalb kein moralisches Gut, weil es überhaupt keine moralischen Güter gibt: Nihilismus also anstelle von religiösem Vertrauen auf letzte Werte. Und in der Tat, Mills Argument taugt nicht, um den Amoralisten zur Raison zu bringen. Der Amoralist kann Mill in allen psychologischen Behauptungen beipflichten und immer noch fragen: Warum sollte ich moralisch sein und das allgemeine Glück zu vermehren suchen?

Ich denke nicht, dass Mill gemeint hat, dem Amoralisten mit zwingenden Argumenten beikommen zu können. Unsere Passage enthält jedenfalls keine Ressourcen für diese Auseinandersetzung - so wenig wie die übrigen Teile der kleinen Schrift. Mill wendet sich an Leser, die nach der richtigen Moral suchen, nicht an Leser, die allererst auf die Suche nach der richtigen Moral gebracht werden müssen. ${ }^{34}$ Schon deshalb ist sein Argument kein zwingendes Argument im Sinne der Logik. Sein Argument kann nur funktionieren, wenn folgende Voraussetzung zutrifft, für die er nicht eigens argumentiert:

(D) Es gibt Güter, die um ihrer selbst willen angestrebt werden sollten.

Dass der Standard für diese Güter bei uns Menschen liegt und nicht irgendwo jenseits der Menschheit, das war die andere Voraussetzung (C), die Mill nicht zwingend begründet. Er scheint diese Voraussetzung für selbstverständlich gehalten zu haben. Und in der Tat wirkt die Voraussetzung vernünftig - mehr noch für unser säkulares Zeitalter als schon zu Mills eigener Zeit.

Die beiden Voraussetzungen (C) und (D), die Mill für sein Argument braucht, gehen übrigens Hand in Hand mit erkenntnistheoretischem Optimismus für die Moral. Wenn es Gutes gibt und wenn der Standard für das Gute bei uns Menschen liegt, dann haben wir faire Chancen herauszufinden, worin dieser Standard besteht. Wie können wir das herausfinden? Für einen Empiristen vom Schlage Mills steht fest: Durch 
apriorische Überlegung finden wir gewiss nicht heraus, welche Dinge als Güter erstrebenswert sind - so wenig, wie wir durch apriorische Überlegung herausfinden können, welche Dinge sichtbar oder hörbar sind. ${ }^{35}$ Nun haben wir Augen und Ohren, um das Sichtbare und Hörbare wahrzunehmen; aber wir haben keine Sinnesorgane zur Wahrnehmung des Erstrebenswerten. Trotzdem gibt es einen nicht-apriorischen Weg zur Erkenntnis des Erstrebenswerten. Er beginnt bei sorgfältiger Beobachtung der menschlichen Psyche, führt zu generellen Aussagen, über das, was die Menschen erstreben, und von dort in die Moral.

Dass Mills Übergang in die Moral nicht logisch erzwungen werden kann, haben wir zugegeben. In dieser Hinsicht unterscheidet sich das Erstrebenswerte vom Sichtbaren und Hörbaren. Die Standards für Sicht- und Hörbarkeit hängen auf jeden Fall nur von uns Menschen ab; es wäre eine verrückte Form von Metaphysik, schlechthin sichtbaren Dingen das Wort zu reden, die doch kein Mensch sehen kann - oder zu bestreiten, dass etwas von uns Menschen Gesehenes wirklich sichtbar sei.

Nicht ganz so verrückt sind die entsprechenden Schachzüge beim Erstrebenswerten. Ohne sich in logische Widersprüche zu verwickeln, kann man (z.B. aus religiösen Gründen) an schlechthin erstrebenswerte Dinge glauben, die kein Mensch anstrebt - oder man kann (wie der Nihilist) bestreiten, dass etwas von uns allen Angestrebtes wirklich erstrebenwert sei. Trotzdem wären solche Schachzüge nicht vernünftig. Sie rücken den Gegenstand der Moral zu weit weg von uns - in die unerreichbaren Gefilde der Metaphysik. Wer nach Gütern sucht, die wir Menschen erstreben sollten und deren Wert wir Menschen erkennen können, der ist gut beraten, wenn auch nicht gezwungen, so wie Mill bei uns Menschen anzusetzen.

Dort liegt die einzige Evidenz, die uns bei der Suche nach erstrebenswerten Gütern weiterhelfen kann. ${ }^{36}$ So verstanden, ist der dritte Satz aus unserer Passage weit weniger anstößig, als man beim ersten Lesen meinen mag. Er besagt: Wenn wir überhaupt über das Erstrebenswerte Aufschluss erlangen können, dann durch empirischen Blick auf das, was wir anstreben.

Wie wir gesehen haben, will Mill empirisch herausgefunden haben, dass die Menschen letztlich nach nichts anderem streben als nach Glück. Ob diese psychologische Behauptung (samt weiterer psychologischer Annahmen, die Mill machen muss) überzogen sind, konnten wir hier nicht erörtern. Aber wir haben versucht, Mills Weg von diesen Annahmen in die Moral plausibel zu machen. Wenn unsere Überlegungen triftig waren, dann sind wir mit Mill zu folgendem Ergebnis gelangt: 
[4 $\left.{ }^{\mathrm{p}}\right]$ Das allgemeine Glück ist das einzige Gut.

Oder, in anderen Worten, die meiner Ansicht nach auf dasselbe hinauslaufen:

$\left[5^{\mathrm{p}}\right] \quad$ Nur das allgemeine Glück ist erstrebenswert; das allgemeine Glück sollte der letzte Zweck menschlichen Handelns sein.

Mill zieht aus diesen Ergebnissen einen weiterreichenden Schluss, den wir bislang verschwiegen haben:

Das allgemeine Glück ist das einzige Kriterium der Moral.

Erst mit diesem Satz wäre der Utilitarismus etabliert: eine allumfassende Moral-Lehre, die den gesamten Bereich der Moral auf Abschätzungen des zu erreichenden allgemeinen Glücks zurückführt. ${ }^{37}$ Ergibt sich dieser Alleinvertretungsanspruch aus den Überlegungen, die wir nachzuzeichnen versucht haben? Ich finde nicht. Wenn alle moralischen Fragen auf Fragen nach Zwecken hinausliefen, hätte Mill vielleicht recht. Aber schon die Frage:

Wie sollte ich handeln?

hat andere Facetten als ihr verengtes Gegenstück:

Welche Zwecke sollte ich verfolgen?

Zudem bedrängen uns weit mehr moralische Fragen als die Frage nach dem richtigen Tun. Selbst wenn Mills Gedankengang aus unserer Passage plausibel sein sollte, führt von dort kein plausibler Weg zu den überzogenen Ansprüchen der Utilitaristen, alle Fragen der Moral im Griff zu haben. Zum Glück nicht! 


\section{Anmerkungen}

1 Dieser Aufsatz ging aus einem Vortrag hervor, den ich im Habilitationskolloquium an der Georg-August-Universität in Göttingen gehalten habe. Dank an Wolfgang Carl, Konrad Cramer, Felix Mühlhölzer, Günther Patzig und Thomas Schmidt für wertvolle Anregungen und konstruktive Kritik.

2 Um der Einheitlichkeit willen werde ich alle Vorkommnisse der englischen Ausdrücke "desirable, to desire" etc. immer durch deutsche Wörter wie "erstrebenswert, anstreben, streben nach" etc. wiedergeben.

3 Positive Bewertungen von Dingen oder Zuständen haben zwar nicht unmittelbar mit Fragen des richtigen Handelns zu tun; aber sie ziehen handlungsrelevante moralische Urteile nach sich. (Wenn sie das nicht täten, so hingen sie ohne Bodenhaftung in der Luft herum). Dieser Zusammenhang zwischen der axiologischen Bewertung von Dingen und der normativen Beurteilung von Handlungen ist nicht unproblematisch. (Siehe z.B. Mandelbaum [TMIi]:231n47). Ich werde den Zusammenhang hier nicht hinterfragen, weil fast alle Utilitaristen ihn wie selbstverständlich voraussetzen. Dass sich der Zusammenhang umkehren lässt, dass also normative Urteile über Handlungen immer auf axiologische Urteile zurückgeführt werden müssen, werde ich nicht voraussetzen, sondern im letzten Abschnitt bestreiten.

4 Mill ließ sich fast zwei Jahre Zeit, bevor er den bereits 1859 im ersten Entwurf fertiggestellten Text veröffentlichte; im Jahr 1860 hat er den Text gründlich überarbeitet, siehe Robson [TI]:cxxiv/cxxv.

5 In der monumentalen Mill-Ausgabe [CWoJ] wird dieser Brief an zwei Stellen dokumentiert, siehe Mill [LLoJ]:1391n7 und Robson [TI]:cxxvi. Ob Gomperz den Brief auf Deutsch oder Englisch verfasst hat und ob der Brief auf den 18.3.1868 (so Robson [TI]:cxxvi) oder auf den 26.3.1868 (siehe Mill [LLoJ]:1391n7) datiert ist, lässt sich anhand der Mill-Ausgabe nicht ermitteln.

6 In der von Gomperz herausgegebenen Übersetzung schreibt Wahrmund: "Der einzige Beweis, welcher dafür gegeben werden kann, dass ein Gegenstand sichtbar sei, besteht darin, dass man ihn wirklich sieht; der einzige Beweis, dass ein Schall hörbar ist, besteht darin, dass man ihn wirklich hört; und eben dasselbe findet auch rücksichtlich der übrigen Quellen unserer Erfahrung statt. In derselben Weise nun, denke ich, besteht auch der einzige Beweis, den man dafür vorbringen kann, dass irgend Etwas wünschenswert sei, darin, dass die Menschen dasselbe wirklich wünschen" (Mill ([NP]:166, meine Schreibweise). Hundert Jahre später übersetzt Birnbacher den letzten dieser Sätze so: "Ebenso wird der einzige Beweis dafür, dass etwas wünschenswert [desirable] ist, der sein, dass die Menschen es tatsächlich wünschen" (Mill ([dU]:60/1; meine Hervorhebung, meine Schreibweise). Durch den eckig eingeklammerten Zusatz macht Birnbacher den Stolperstein besonders sichtbar, statt ihn (Mills Bitte an seine Vorgänger folgend) stillschweigend zu beseitigen. Andererseits könnte man Birnbachers Verwendung der Futurform des Verbs als einen Verbesserungsvorschlag ansehen, der darauf hindeuten soll, dass man auf den eigentlichen Beweis noch zu warten hat. Falls Birnbacher die Futurform so gemeint hat, passt seine Übersetzung gut zu den Grundlinien meiner Interpretation.

7 Die eckig eingeklammerten Satznummern entsprechen der Numerierung, die ich oben in die zitierte Passage eingearbeitet habe.

8 Mill setzt unsere Passage so fort: "But it [i.e., happiness - O.M.] has not, by this alone, proved itself to be the sole criterion. To do that, it would seem, by the same rule, necessary to show, not only that people desire happiness, but that they never desire anything else" (Mill [U]:234 (IV, §4); meine Hervorhebung). Die zurückhaltende Formulierung "it would seem" wählt Mill nicht, weil er den parallelen Gedankenstrang am Ende doch nicht in Anspruch nehmen möchte, sondern weil dessen Voraussetzung auf den 
ersten Blick unplausibel ist und daher längerer Entfaltung bedarf. Mill entfaltet die Voraussetzung denn auch in den Absätzen, die unserem Zitat folgen (Mill [U]:235-237 (IV,§4-7)). Und in der dann folgenden Zusammenfassung zieht er den Schluss, den er bereits angekündigt hat, ohne allerdings die Details aus unserer Passage parallel zu wiederholen (Mill [U]:237 (IV, §8-9)).

9 Durch ein hochgestelltes $\mathrm{P}$ an irgendeiner Satznummer [np] werde ich andeuten, dass der fragliche Satz das ambitionierte Gegenstück des Satzes [n] aus unserer Passage darstellt. Dies ambitionierte Gegenstück [np] muss nicht unbedingt in Mills Text vorkommen, da Mill den ambitionierten Parallelstrang seines Gedankengangs nicht im Detail durchdekliniert.

10 Siehe Moore [PE]:118/19 (§40). Eine ausführliche Darstellung und Kritik dessen, was Moore aus Mills Text herausgelesen haben will, bietet Hall [PoUi]:146-154.

11 Siehe Moore [PE]:66-68 (§13).

12 Selbst wenn die Parallele bis in die Semantik reichen sollte, spräche dies nicht dafür, das Erstrebenswerte mit dem Angestrebten zu identifizieren. Es spräche nur dafür, das Erstrebenswerte mit dem zu identifizieren, was angestrebt werden kann. (Wenn wir diesen Einwand allerdings im Zusammenhang mit Mills ambitionierteren Gedankengang wiederholen wollen, den wir im Abschnitt II vorgeführt haben, dann verflüchtigt sich die Kraft des Einwandes. Denn in jenem ambitionierteren Gedankengang kommt Mill zu dem Ergebnis: "Nur das Glück wird angestrebt". Das könnte man so verstehen, als wolle Mill sagen: "Menschen können nichts anderes anstreben als das Glück". Und dann könnte die angebliche semantische Parallele zwischen "sichtbar" und "erstrebenswert" wirklich dafür sorgen, dass gilt: "Nichts anderes als das Glück ist erstrebenswert").

13 Siehe Mill [U]:207/8 (I, §5) und 234 (IV, §1).

14 Jedenfalls an einer einzigen Stelle in unserer Passage. Im parallelen, ambitionierteren Gedankengang, von dem im Abschnitt II die Rede war, vollzieht er den fraglichen Übergang natürlich ein zweites Mal. (Siehe Fußnote 32 unten in Abschnitt VII).

15 Sätze mit negativer Satznummer finden sich in Mills Text nicht.

16 Und im Lichte des ambitionierteren Parallelstranges seiner Überlegung wäre Mill dann auch auf folgende Behauptung festgelegt: [-2p] "Jeder erstrebt nichts anderes als das allgemeine Glück". Es ist klar, dass Mill dieser optimistischen Behauptung nicht zugestimmt hätte.

17 Siehe z.B. Mill [A]:149.

18 Der Satz "Jedes Schwein erstrebt das Futter aller Schweine" bedeutet genauer: "Jedes Schwein strebt danach, dass es das Futter aller Schweine fressen kann". Aber der Satz "Jedes Schwein erstrebt die Lust aller Schweine" hat eine andere Struktur: "Jedes Schwein strebt danach, dass alle Schweine Lust empfinden". Wer Schweinen solche feinen strukturellen Unterschiede nicht zugestehen mag, sollte sich daran erinnern, dass Bradley einer langen Tradition gefolgt ist, als er diese Tiere ins Spiel brachte: Seit Epikur werden Anhänger einer hedonistischen Wertlehre von ihren Gegnern als Schweine bezeichnet.

19 Zur Terminologie: Mit dem Ausdruck "Werthaltung einer Person" möchte ich nicht die Meinung der Person bezeichnen, dass das und das ein Wert sei, sondern seine Neigung, den fraglichen Wert wirklich anzustreben. Nur im Fall rationaler Personen gehen beide Dinge Hand in Hand.

20 Dass Mill im Weg von [2] nach [3] keine schwierigen Probleme sah (also z.B. keinen Übergang vom Beschreiben zum Werten), sondern einen direkten Schluss sozusagen per Summenbildung, hat er am Ende eines Briefs vom 13.6.1868 klargestellt: "I merely meant in this particular sentence to argue that since A's happiness is a good, B's a good, C's a good, \&c., the sum of all these goods must be a good" ([LLoJ]:1414; meine Hervorhebung). Dass er hier nicht wiederholt, für wen das jeweilige Gut ein Gut ist, hat meiner Ansicht nach stilistische Gründe. Er lässt keinen Zweifel, dass er sich 
auf den Satz aus unserer Passage bezieht, in dem diese Zusätze ausdrücklich zu finden sind.

21 Hier müssen wir uns gegen ein Missverständnis wappnen. Die Behauptung [3*] bedeutet nicht: "Für jeden Menschen X gilt: Es ist ein Gut für X, dass alle Menschen glücklich sind". Denn das wäre wieder eine Beschreibung der tatsächlichen Werthaltungen der Menschen, die falsch ist und die Mill auch für falsch hält. (Mill stellt das in einem Brief vom 13.6.1868 klar: "[...] when I said that the general happiness is a good to the aggregate of all persons I did not mean that every human being's happiness is a good to every other human being; though I think, in a good state of society and education it would be so" ([LLoJ]:1414)). Meiner Ansicht nach lässt sich die beschreibende Rede vom "Gut für eine Gruppe" im allgemeinen nicht in eine Summe von Beschreibungen der Güter für jeden einzelnen zerlegen. (In dieser Hinsicht ist das Tibet-Beispiel zu einfach). Wie wir im nächsten Abschnitt sehen werden, kommen bei der Rede von Gütern für eine Gruppe objektive Zusatzbedingungen ins Spiel.

22 Wir wollen hier davon absehen, dass die Lottogesellschaft üblicherweise einen Teil der Einsätze abschöpft.

23 Mill [U]:233 (III, §11).

24 Mill [U]:235 (IV, §5).

25 Mill [U]:235-237 (IV, §5-7).

26 Diese Voraussetzung ist selber wertneutral; man muss nicht schon Utilitarist sein, um sie für wahr zu halten. Zwar habe ich oben auf Mills dezidiert utilitaristische Konzeption der Tugend zurückgegriffen (siehe dazu Mandelbaum [oIMU]:43) - aber nicht um die Voraussetzung zu begründen, sondern um zu begründen, dass Mill diese Voraussetzung unterschreibt. Um die Voraussetzung inhaltlich $\mathrm{zu}$ begründen, müsste man psychologische Untersuchungen anstellen.

27 An dieser Stelle werden Mills Überlegungen aus dem zweiten Kapitel wichtig, in dem er Glückszuständen sinnlicher Herkunft geringere Qualität zuschreibt als z.B. intellektuellen Glückszuständen oder Glückszuständen, die auf moralischen Gefühlen beruhen (Mill [U]:210-212 (II, §4-6)). In einem früheren Aufsatz beschränkt Mill denselben Punkt zielgenauer auf die Moral und spricht in diesem Zusammenhang von einem "state of mind, without which [one's] own enjoyment of life can be but poor and scanty" ([RoBP]:15). Siehe auch [U]:215, 217, 231 (II, §13, §14; III, §10).

28 Zugegebenermaßen sagt Mill an der fraglichen Stelle in unserer Passage nur, dass das Glück ein Gut ist; ob er das allgemeine Glück oder das Glück des einzelnen im Auge hat, lässt er offen. Ich habe mich entschlossen, seine Aussage in Richtung auf das allgemeine Glück zu verschärfen, weil Mill im ambitionierten Parallelstrang seines Gedankenganges nicht sagen darf: "Das Glück des einzelnen ist das einzige Gut". Er muss sagen: [4p] "Das allgemeine Glück ist das einzige Gut". Und erst nachdem er diese Wertung erreicht hat, kann er irgendwann später ableiten: "Das Glück des einzelnen ist ein Gut". Dieser Schluss ergibt sich aus [4p], wenn man hinzunimmt, dass das Glück des einzelnen ein Bestandteil des kollektiven Glücks ist. Allerdings findet sich solch ein Schluss nirgends bei Mill. Immerhin erlaubt er die Rede von Teilen des Glücks, wenn auch in einem anderen Zusammenhang: "Happiness is not an abstract idea, but a concrete whole; and these are some of its parts" (Mill [U]:236 (IV, §6)). Fest steht, dass er den Wert des Glücks des einzelnen, wenn überhaupt, dann nur irgendwo außerhalb des parallel geführten Gedankengangs erreichen kann.

29 Jedenfalls begründet Hume die Sein/Sollen-Schranke bloß unter Verweis auf gescheiterte Schlüsse vom Sein zum Sollen, siehe [ToHN]:469.

30 D.h. Mills ethische Lehre lässt sich mit der genannten Beschreibung der indischen Meditationsgemeinschaft vereinbaren; Mills Argument für diese Lehre lässt sich mit dieser Beschreibung natürlich nicht vereinbaren; denn sie widerspricht seiner psychologischen Prämisse, der zufolge alle Menschen nach Glück streben. 
31 Unsere augenblickliche Überlegung hat eine gewisse Verwandtschaft mit dem sogenannten Moore-Paradox, siehe Moore [RToD]:175/6.

32 Ich werde die beiden Fragen im folgenden nicht strikt auseinanderhalten. Die erste Frage betrifft die Triftigkeit unserer Passage; die zweite Frage betrifft die Triftigkeit des ambitionierten Parallelstranges der Überlegungen Mills, auf den ich im Abschnitt II hingewiesen habe. Wenn unsere bisherigen Ausführungen und Mills psychologische Annahmen stimmen, dann wäre Mill in seinem Parallelstrang bislang zu folgendem beschreibenden Satz vorgedrungen: [3p] "Das Glück ist das einzige Gut für die Gesamtheit der Menschen". Für Mills Zwecke würde es genügen, wenn er von diesem Punkt in die Moral vordringen könnte, selbst wenn derselbe Übergang aus unserer Passage nicht gangbar wäre.

33 Obwohl er sich hierüber in Utilitarianism nicht ausführlich äußert (siehe Mill [U]:229 (III, §6)), steht außer Zweifel, dass Mill Behauptungen für richtig hielt, die ungefähr in die Richtung von (C) laufen. So sagt er an anderer Stelle, dass Sir Hamiltons Lehren zu einer religiösen Sicht geführt hätten "which I hold to be profoundly immoral - that it is our duty to bow down in worship before a Being whose moral attributes are affirmed to be unknowable by us, and to be perhaps extremely different from those which, when we are speaking of our fellow creatures, we call by the same names" ([A]:176). Und in einem Aufsatz über die Nützlichkeit der Religion heißt es: "[...] there is a very real evil consequent on ascribing a supernatural origin to the received maxims of morality. [...] Belief, then, in the supernatural $[\ldots]$ cannot be considered to be any longer required, either for enabling us to know what is right and wrong in social morality, or for supplying us with motives" ([UoR]:417; vergl. auch [UoR]:405). Diese Zitate bieten natürlich kein unabhängiges Argument zugunsten von (C), da sich Mill hier bereits auf dem Boden seiner eigenen Moral-Lehre bewegt und da wir (C) zur Vervollständigung seines Beweises zugunsten dieser Lehre ins Spiel gebracht haben.

34 Für diese Interpretation spricht u.a. der einzige Satz aus unserer Passage, den ich eingangs nicht mit abgedruckt habe: "If the end which the utilitarian doctrine proposes to itself were not, in theory and in practice, acknowledged to be an end, nothing could ever convince any person that it was so" (meine Hervorhebung); trotz anderer Stoßrichtung bei Mill dürfte dieser Satz Amoralisten von der Betrachtung ausschließen. Das bedeutet nicht, dass Mill dem Amoralisten nichts entgegenzusetzen hätte. Er könnte den Amoralisten an die Hand nehmen und ihn erleben lassen, dass Tugend einen unverzichtbaren Teil des glücklichen Lebens ausmacht.

35 Siehe Mill [U]:234 (IV, §1).

36 Einen ähnlichen Punkt macht Brandt [ET]:262. Auf einer sehr verwandten Idee beruht einer der scharfsinnigsten Beweisversuche des Utilitarismus aus dem 20. Jahrhundert: Harsanyi leitet die (präferenz-) utilitaristische Maximierungsformel aus den Rationalitätspostulaten der Entscheidungstheorie und einer einzigen weiteren Annahme ab. Diese zusätzliche Annahme ist zwar nicht moralisch neutral, aber sie ist äußerst schwach und erinnert an Mills Voraussetzungen (C) und (D). Sie besagt ungefähr: "Wenn mindestens ein Mensch die Option I über Option II vorzieht, während kein einziger Mensch II über I vorzieht, dann ist I besser als II" (siehe Harsanyi [MToR]:48/9, Axiom 3). Wer diese Voraussetzung ablehnt, ist entweder Nihilist - oder er glaubt an Werte, die von Menschen ganz unabhängig sind. In dieser Hinsicht ähneln sich Mills und Harsanyis Beweis. Doch anders als Mills Beweis kommt Harsanyis Beweis ohne hedonistische Psychologie aus; das kann man als Vorteil sehen (Harsanyi [MToR]:54).

37 Siehe Mill [U]:205/6, 214 (I, §1-2; II, §10). Es besteht eine leichte Spannung zwischen diesen Passagen und späteren, moderaten Formulierungen, siehe Mill [U]:221 (II, §20-21). 


\section{Literatur}

Bradley, F.H. [ES]: Ethical studies. (Oxford: Oxford University Press, second edition, 1927). [Erschien zuerst 1876].

Brandt, Richard B. [ET]: Ethical theory. The problems of normative and critical ethics. (Englewood Cliffs: Prentice-Hall, 1959).

Hall, Everett W. [PoUi]: "The "Proof" of utility in Bentham and Mill". In Schneewind (ed) [M]:145-178. [Erschien zuerst 1949].

Harsanyi, John C. [MToR]: "Morality and the theory of rational behavior". In Sen / Williams (eds) [Ub]:39-62. [Erschien zuerst 1977].

Hume, David [ToHN]: A Treatise of human nature: Being an attempt to introduce the experimental method of reasoning into moral subjects. (L. A. Selby-Bigge (ed); Oxford: Clarendon Press, 1888). [Erschien zuerst 1739].

Mandelbaum, Maurice [oIMU]: "On interpreting Mill's Utilitarianism". Journal of the History of Philosophy VI (1968), pp. 35-46.

Mandelbaum, Maurice [TMIi]: "Two moot issues in Mill's Utilitarianism". In Schneewind (ed) [M]:206-233.

Mill, John Stuart [A]: Autobiography. (Currin V. Shields (ed); Indianapolis: Bobbs-Merill, 1957). [Erschien zuerst 1873].

Mill, John Stuart [CWoJ]: Collected works of John Stuart Mill. (F.E.L. Priestley (ed); Toronto: University of Toronto Press, 1963ff).

Mill, John Stuart [dU]: Der Utilitarismus. (= Mill [U], deutsche Übersetzung von Dieter Birnbacher; Stuttgart: Reclam, 1976).

Mill, John Stuart [EoER]: Essays on ethics, religion and society. (J.M. Robson (ed); Toronto: University of Toronto Press, 1969). (= Mill $[\mathrm{CWoJ}] / \mathrm{X})$.

Mill, John Stuart [GW]/I: Gesammelte Werke. Autorisierte Übersetzung. Erster Band. (Theodor Gomperz (ed); Leipzig: Fues's Verlag, 1869).

Mill, John Stuart [LLoJ]: The later letters of John Stuart Mill: 1849-1873. (Francis E. Mineka / Dwight N. Lindley (eds); Toronto: University of Toronto Press, 1972, four volumes). (= Mill [CWoJ]/XIV-XVII). 
Mill, John Stuart [NP]: Das Nützlichkeits-Princip. (= Mill [U], deutsche Übersetzung von A. Wahrmund). In Mill [GW]/I:125-201).

Mill, John Stuart [RoBP]: "Remarks on Bentham's philosophy". In Mill [EoER]:3-18. [Erschien zuerst 1833].

Mill, John Stuart [U]: Utilitarianism. In Mill [EoER]:203-259. [Erschien zuerst 1861. Ich zitiere die Seitenzahlen aus der angeführten Ausgabe und füge in Klammern römische Kapitelnummern hinzu, gefolgt von der Nummer des fraglichen Absatzes. Z.B. bezeichnet "IV, §2" den zweiten Absatz des vierten Kapitels].

Mill, John Stuart [UoR]: "Utility of religion". In Mill [EoER]:403-428. [Erschien zuerst posthum 1874].

Moore, George Edward [PE]: Principia ethica. (Cambridge: Cambridge University Press, revised edition, 1993). [Erschien zuerst 1903].

Moore, George Edward [PP]: Philosophical papers. (London: George Allen \& Unwin, 1959).

Moore, George Edward [RToD]: "Russell's 'Theory of Descriptions"". In Moore [PP]:151-195. [Erschien zuerst 1944].

Robson, J.M. [TI]: "Textual introduction". In Mill [EoER]:cxv-cxxxix.

Schneewind, J.B. (ed) [M]: Mill: A collection of critical essays. (London: MacMillan, 1968).

Sen, Amartya / Williams, Bernard (eds) [Ub]: Utilitarianism and beyond. (Cambridge: Cambridge University Press, 1982). 